\title{
Modeling Line Emission from Disk Winds
}

\author{
Patrick B. Hall ${ }^{1}$ and Laura S. Chajet ${ }^{1}$ \\ ${ }^{1}$ Department of Physics \& Astronomy, York University, Toronto, Ontario, Canada \\ Email: phall, lchajet@yorku.ca
}

Keywords. galaxies: active, quasars: general, quasars: emission lines

Murray \& Chiang (1997) developed a model wherein broad emission lines come from the optically thick base of a rotating, outwardly accelerating wind at the surface of an accretion disk. Photons preferentially escape radially in such a wind, explaining why broad emission lines are usually single-peaked. Less well understood are the observed shifts of emission-line peaks (from $1000 \mathrm{~km} \mathrm{~s}^{-1}$ redshifted to $2500 \mathrm{~km} \mathrm{~s}^{-1}$ blueshifted in C IV, with an average $800 \mathrm{~km} \mathrm{~s}^{-1}$ blueshift). The Murray \& Chiang disk wind model produces blueshifts an order of magnitude smaller. However, their model makes use of a number of simplifying approximations. We have extended their analysis by making many fewer assumptions about the velocity field of the wind. Our goal is to find disk wind parameters that can reproduce observed emission-line blueshifts.

Figure 1 shows normalized C IV line profiles for a $L_{\mathrm{UV}}=10^{46} \mathrm{erg} \mathrm{s}^{-1}$ quasar with $M_{\mathrm{BH}}=$ $10^{8} M_{\odot}$ for six values of the disk inclincation $i$, roughly evenly spaced in solid angle between $i=0^{\circ}$ and $i=60^{\circ}$. These profiles are for a model in which the wind launch velocity at each radius equals the Keplerian velocity at that radius, the streamlines are straight (but nonradial) with constant poloidal angle $\lambda$, and a CLOUDY-derived source function is used. We find that such winds can produce blueshifts as large as observed when the streamlines are more vertical than horizontal $\left(\lambda>45^{\circ}\right)$ and the quasars are viewed at $i<45^{\circ}$. Note that larger blueshifts are observed for disk winds viewed closer to face-on, even when the winds are nearly equatorial.
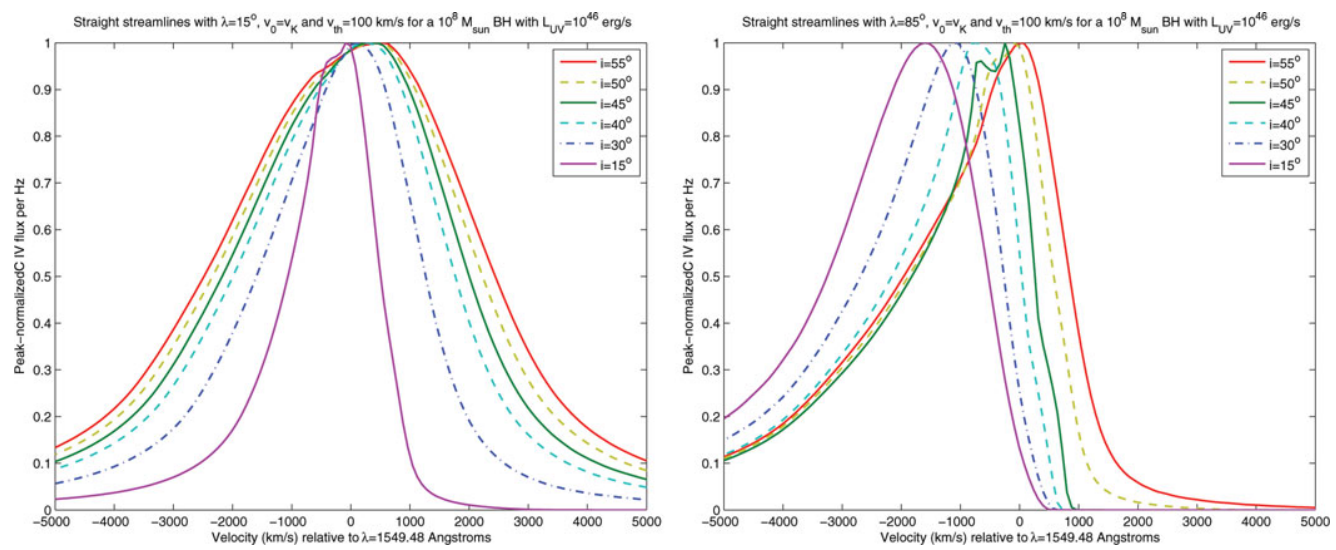

Figure 1. Normalized C IV emission-line profiles. Negative velocities denote blueshifts towards the observer. Left: For nearly equatorial straight streamlines $\left(\lambda=15^{\circ}\right), \mathrm{C}$ IV is blueshifted only when viewed from the most pole-on sightline. Increased rotation along the line of sight increases the FWHM at larger $i$, where the disk is seen closer to edge-on. Right: For nearly vertical straight streamlines $\left(\lambda=85^{\circ}\right)$, a blue wing is seen in C IV at all inclination angles, blueshifts are seen for two-thirds of the solid angle with $i<60^{\circ}$, and the FWHM varies only weakly with $i$.

\section{Reference}

Murray, N. \& Chiang, J. 1997, ApJ, 474, 91 